\title{
Produção científica da enfermagem sobre morte/ morrer em unidades de terapia intensiva
}

Recebido em: 08/07/2013

Aceito em: 25/11/2014
Anderson Souza Almeida

Edison Luiz Devos Barlem²

Jamila Geri Tomaschewski-Barlem ${ }^{3}$

Valéria Lerch Lunardi

Resumo: conhecer a produção científica nacional de enfermagem acerca da morte/morrer em ambientes de Unidade de Terapia Intensiva. Revisão integrativa realizada através dos descritores morte, unidades de terapia intensiva e enfermagem, entre os anos de 2005 e 2011 , obtendo-se um corpus de 14 artigos. RESULTADOS: foram construídas três categorias: prática como objeto de reflexão e mudança; preparo profissional e instrumentalização; desafios éticos frente o processo de morte/morrer. A produção científica sobre morte/morrer deve ser redirecionada, ultrapassando os limites investigativos baseados meramente na busca da percepção dos significados e dos sentimentos.

Descritores: Morte; Unidades de Terapia Intensiva; Enfermagem.

\section{Scientific production from the nursing about death / dying in intensive care units}

Abstract: Know the scientific production of nursing about death / dying in environments Intensive Care Unit. METHODOLOGY: integrative review conducted by the descriptors death, intensive care units and nursing, between the years 2005 and 2011, resulting in a corpus of 14 articles. RESULTS: Three categories were constructed: practice as an object of reflection and change, professional preparation and instrumentation; ethical challenges facing the process of death / dying. CONCLUSION: the scientific literature on death / dying must be redirected, overstepping the bounds investigative search based merely on the perception of the meanings and feelings.

Descriptors: Death; Intensive Care Units; Nursing.

\section{Producción científica de enfermería de la muerte/morir en unidades de cuidados intensivos}

Resumen: Conocer la producción científica de enfermería sobre la muerte/morir en entornos Unidad de Cuidados Intensivos. METODOLOGÍA: revisión integradora realizada por los descriptores muerte, unidades de cuidados intensivos y enfermería, entre los años 2005 y 2011 , dando lugar a un corpus de 14 artículos. RESULTADOS: Tres categorías se construyeron: la práctica como objeto de reflexión y cambio, la preparación profesional y la instrumentación, los desafíos éticos que enfrenta el proceso de la muerte / la muerte. CONCLUSIÓN: la literatura científica sobre la muerte / muerte debe ser redirigida, sobrepasando los límites de la búsqueda investigativa basada meramente en la percepción de los sentidos y los sentimientos.

Descriptores: Muerte; Unidades de Cuidados Intensivos; Enfermería.

\section{INTRODUÇÃO}

$\mathrm{D}$ o ponto de vista biológico, no ciclo de vida humano, a morte é apenas mais uma etapa a ser cumprida a cada ser vivo. Contudo, considerando a condição humana do homem, e a certeza absoluta de sua mortalidade, o tema morte é um assunto difícil de ser abordado. Atrelado a essas questões, verificam-se dimensões éticas, culturais, religiosas e socioambientais, demonstrando a importância de uma atenção especial ao assunto, principalmente entre os trabalhadores de enfermagem, tanto na perspectiva da formação acadêmica como da atuação profissional.

Historicamente, o tema morte sofreu mudanças na sua abordagem, principalmente, com os avanços tecnológicos que proporcionaram recursos capazes de mudar o objetivo da assistência prestada aos doentes. $O$ surgimento das unidades de terapia intensiva (UTIs) alavancou novas mudanças, destacando-se a transformação no foco do tratamento que passou a ser embasado na cura e a morte passou a ser vista mais do que nunca como um insucesso biomédico. Assim, a própria duração da vida em situações de morte e morrer, termo que define o esgotamento das possibilidades de resgate das condições de saúde do paciente, passou a ser prolongada, principalmente em locais específicos como as UTI (1-2).

Na sociedade contemporânea, cada vez mais a morte em ambientes hospitalares acontece nas UTI, onde o paciente em processo de morte morrer, em muitos casos, recebe prioritariamente dispositivos invasivos ao invés de conforto e analgesia ${ }^{(3)}$. Além disso, o paciente em processo de morte morrer apresenta em curto prazo uma probabilidade alta de mortalidade devido a impossibilidade de reversão do quadro patológico, situação que pode gerar ambíguas situações de conflito e vínculo tanto para a família como para a equipe, onde em sua maioria a aceitação da morte é bastante conturbada ${ }^{(4-6)}$.

Assim, identificando a necessidade de conhecer a produção científica nacional sobre a morte em ambientes de UTI, verificando os avanços alcançados e as dificuldades ainda existentes para os profissionais da saúde, objetivou-se: conhecer a produção científica nacional de enfermagem acerca do tema morte/morrer em ambientes de UTI.

\section{METODOLOGIA}

Trata-se de uma revisão integrativa, abordagem que permite na prática a busca, avaliação critica e síntese dos temas investigados ${ }^{(7)}$. Foram seguidas as seguintes etapas: formulação e identificação do problema; coleta de dados; avaliação dos dados; análise e interpretação dos dados coletados; apresentação dos resultados ${ }^{(8)}$.

Na primeira etapa, formulação e identificação do problema, foi realizado primeiramente o aprofundamento teórico sobre o tema morte/morrer, o que permitiu definir o problema de pesquisa: qual a produção científica nacional acerca da morte/morrer na enfermagem?

Para etapa de coleta de dados, foram utilizadas as base de dados Literatura Latino-Americana e do Caribe em Ciências 
da Saúde (LILACS), Índice Bibliográfico Espanhol de Ciências de Saúde (IBECS) e a biblioteca Scientific Electronic Library Online (SCIELO). Os critérios de inclusão das publicações definidos para esta revisão integrativa foram: artigos de pesquisa; desenvolvido por autores brasileiros; disponíveis on line na íntegra; responder ao problema formulado; publicados em periódicos de enfermagem entre 2005 e 2011; apresentados em língua portuguesa, inglesa ou espanhola; abordar a temática terminalidade e UTI.

Utilizaram-se como descritores: "enfermagem", "morte" e "unidade de terapia intensiva". O operador boleano utilizado foi AND. A busca foi realizada pelo acesso on-line, no mês de março de 2012. Para etapa de avaliação dos dados, os 38 artigos encontrados mediante a aplicação dos critérios estabelecidos foram avaliados quanto a aderência temática e pertinência, selecionando-se quatorze artigos para a realização desta revisão integrativa.

Na etapa de análise e interpretação dos dados, foi elaborado um instrumento contemplando as seguintes variáveis: título da publicação, periódico, ano de publicação, tipo de artigo, autor(es), objetivo do estudo e enfoque principal no artigo selecionado. O processo de análise dos dados ocorreu através de confecção de quadros com as variáveis envolvidas nos estudos e com os enfoques utilizados.

Após, utilizou-se para formular a etapa de formulação dos resultados a Análise Textual Discursiva, técnica de análise que é desenvolvida através de um processo integrado de análise e síntese, baseada em uma leitura exaustiva e aprofundada, descrevendo e interpretando significados e fenômenos, organizando os achados em categorias ${ }^{(9)}$. A última etapa da revisão, apresentação dos resultados, é demonstrada no capítulo a seguir:

\section{RESULTADOS}

Em relação aos periódicos, os quatorze artigos selecionados estavam distribuídos entre os seguintes periódicos: Revista da Escola de Enfermagem da USP (três artigos), Revista Mineira de Enfermagem (três artigos), Acta Paulista de Enfermagem (dois artigos), Revista de Enfermagem do Nordeste (dois artigos), Ciência, Cuidado e Saúde (um artigo), Revista Brasileira de enfermagem (um artigo), Revista Gaúcha de Enfermagem (um artigo) e Revista Latino- America de Enfermagem (um artigo). Quanto ao tipo de publicação, todos os artigos eram pesquisas originais.

O número de autores por artigo sofreu uma variação entre um e seis, totalizando 41 (quarenta e um) articulistas, dos quais somente três estavam presentes em mais de uma publicação. Assim, observou-se que Gutierrez BAO e Ciampone MHT foram co-autores de dois artigos, enquanto Lunardi VL foi autora de outros dois artigos com co-autores diferentes.

Quanto aos objetivos propostos pelas publicações, observou-se que a maioria dos autores utilizou-se de estudos exploratórios, buscando conhecer, identificar, caracterizar ou verificar as percepções dos enfermeiros acerca da morte e morrer no contexto da UTI, percebendo-se assim, uma acentuada homogeneidade entre os objetivos identificados. O quadro 1 contempla as variáveis do estudo.

\begin{tabular}{|cccccc}
\hline Título do artigo & Periódico & Ano & Tipo & Autor & Objetivos \\
\hline $\begin{array}{l}\text { Identificação das fases do } \\
\text { processo de morrer pelos } \\
\text { profissionais de Enfermagem }\end{array}$ & $\begin{array}{c}\text { Paulista de } \\
\text { Enfermagem }\end{array}$ & 2006 & Pesquisa & $\begin{array}{c}\text { Susaki TT } \\
\text { Silva MJP da } \\
\text { Possari JF }\end{array}$ & $\begin{array}{l}\text { Verificar se o enfermeiro consegue } \\
\text { identificar as cinco fases do processo } \\
\text { de morrer, descritas por Elizabeth } \\
\text { Kübler-Ross. }\end{array}$ \\
\hline
\end{tabular}

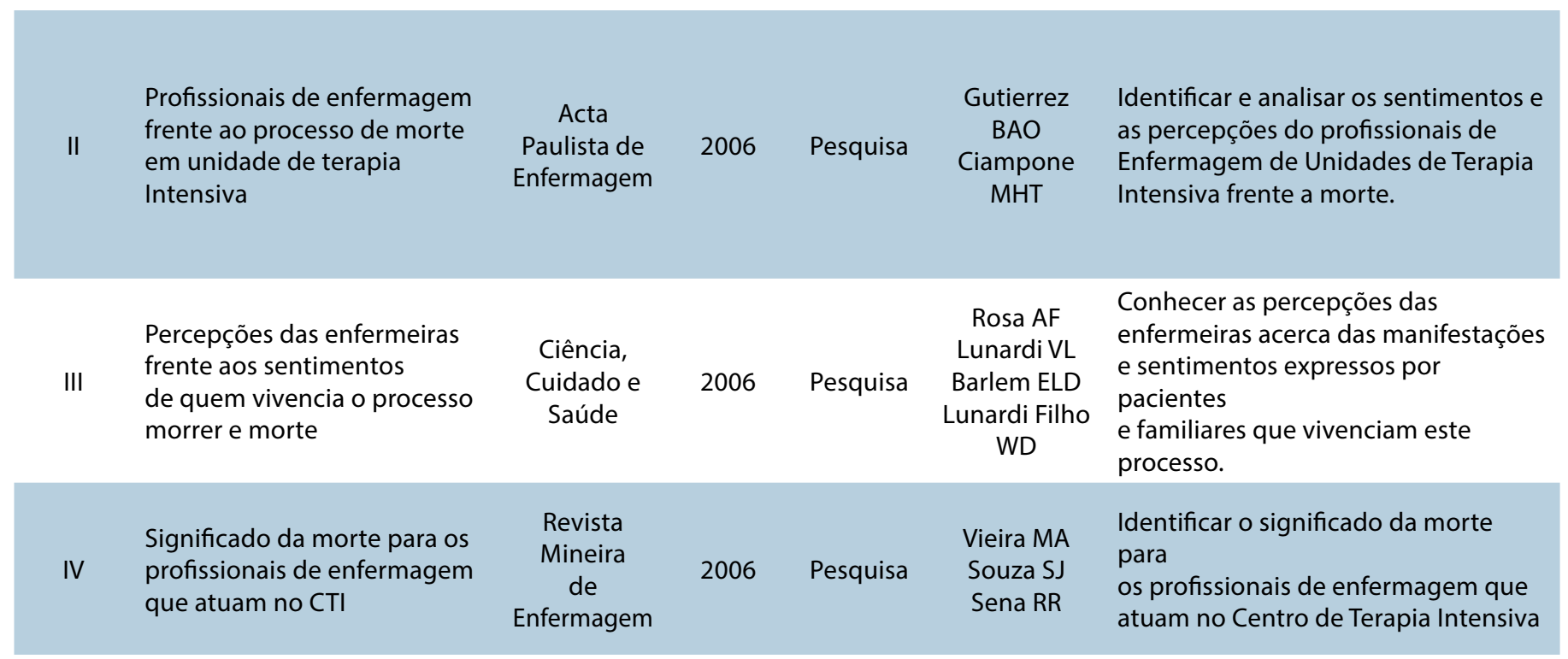




\begin{tabular}{|c|c|c|c|c|c|c|}
\hline V & $\begin{array}{l}\text { A morte em unidade de } \\
\text { terapia } \\
\text { intensiva: percepções do } \\
\text { Enfermeiro }\end{array}$ & $\begin{array}{l}\text { Revista de } \\
\text { Enfermagem } \\
\text { do } \\
\text { Nordeste }\end{array}$ & 2006 & Pesquisa & $\begin{array}{l}\text { Fernandes } \\
\text { MEN } \\
\text { Fernandes } \\
\text { AFC } \\
\text { Albuquerque } \\
\text { ALP } \\
\text { Mota MLS }\end{array}$ & $\begin{array}{l}\text { identificar a compreensão do } \\
\text { enfermeiro que trabalha em Unidade } \\
\text { de Terapia Intensiva acerca do } \\
\text { processo morte e o vivenciar da } \\
\text { morte do paciente. }\end{array}$ \\
\hline VI & $\begin{array}{l}\text { A significação do óbito } \\
\text { hospitalar para enfermeiros } \\
\text { e médicos }\end{array}$ & $\begin{array}{l}\text { Revista de } \\
\text { Enfermagem } \\
\text { do } \\
\text { Nordeste }\end{array}$ & 2006 & Pesquisa & $\begin{array}{l}\text { Nascimento } \\
\text { CAD } \\
\text { Silva ADB } \\
\text { Silva MC } \\
\text { Pereira } \\
\text { MHDM }\end{array}$ & $\begin{array}{l}\text { Identificar as significações sobre a } \\
\text { a qual se materializa o processo } \\
\text { do óbito hospitalar. }\end{array}$ \\
\hline
\end{tabular}

$\begin{array}{llccc}\text { Como os trabalhadores de } & \text { Revista } & & \\ \text { enfermagem enfrentam o } & \text { Brasileira } & \text { de } \\ \text { processo de morrer } & \text { 2007 } & \text { Pesquisa } & \text { Shimizu HE } \\ \text { Enfermagem } & & & \end{array}$

Identificar e analisar as representações e sentimentos vivenciados, os mecanismos de defesa

e as estratégias desenvolvidas pelos trabalhadores de enfermagem no processo de enfrentamento da morte

\begin{tabular}{|c|c|c|c|c|c|}
\hline VIII & $\begin{array}{l}\text { O processo de morrer e a } \\
\text { morte no enfoque dos } \\
\text { profissionais de enfermagem } \\
\text { de UTIs }\end{array}$ & $\begin{array}{l}\text { Revista da } \\
\text { Escola } \\
\text { de } \\
\text { Enfermagem } \\
\text { da USP }\end{array}$ & 2007 & Pesquisa & $\begin{array}{c}\text { Gutierrez } \\
\text { BAO } \\
\text { Ciampone } \\
\text { MHT }\end{array}$ \\
\hline
\end{tabular}

IX $\quad \begin{aligned} & \text { Percepções de enfermeiros } \\ & \text { sobre dilemas éticos } \\ & \text { relacionados a pacientes } \\ & \text { terminais em unidades de } \\ & \text { terapia intensiva }\end{aligned}$

Xntre a vida enferma e a
morte sadia: a ortotanásia na
vivência de enfermeiros em
unidade de terapia intensiva
Revista da Escola de Enfermagem da USP

\section{Identificar as concepções}

relacionadas

ao processo de morrer e à morte no

contexto de trabalho dos

profissionais de enfermagem de UTI.

Conhecer a percepção de enfermeiros sobre dilemas éticos existentes na assistência de enfermagem a pacientes terminais, no contexto da UTI de um hospital geral do municipio de São Paulo e o que é considerado para a tomada de decisão.

$\begin{array}{ccc}\begin{array}{c}\text { Revista } \\ \text { Mineira } \\ \text { de }\end{array} & 2009 \text { Pesquisa } & \begin{array}{c}\text { Bisogno SBC } \\ \text { Quintana AM } \\ \text { Camargo VP }\end{array}\end{array}$

$\begin{array}{ccc}\text { Revista } & & \text { Mattos TDAD } \\ \text { Lineira } & \text { Lange C } \\ \text { de } & \text { Cecagno D } \\ \text { Enfermagem } & \text { Pesquisa } & \text { Amestoy SC } \\ \text { Thofehrn MB } \\ \text { Milbrath VM }\end{array}$

\begin{tabular}{|c|c|c|c|c|c|c|}
\hline XII & $\begin{array}{l}\text { Obstinação terapeutica como } \\
\text { questão ética: Enfermeiras de } \\
\text { unidade de terapia intensiva }\end{array}$ & $\begin{array}{l}\text { Revista } \\
\text { Latino- } \\
\text { Americana } \\
\text { de } \\
\text { Enfermagem }\end{array}$ & 2009 & Pesquisa & $\begin{array}{l}\text { de Carvalho } \\
\text { KK } \\
\text { Lunardi VL }\end{array}$ & $\begin{array}{l}\text { compreender como enfermeiras de } \\
\text { UTIs vem enfrentando a aplicação de } \\
\text { medidas terapêuticas que } \\
\text { reconhecem } \\
\text { como fúteis. }\end{array}$ \\
\hline XIII & $\begin{array}{l}\text { Vivência dos enfermeiros de } \\
\text { unidade de terapia intensiva } \\
\text { frente a morte e morrer }\end{array}$ & $\begin{array}{l}\text { Revista } \\
\text { Gaúcha } \\
\text { de } \\
\text { Enfermagem }\end{array}$ & 2009 & Pesquisa & $\begin{array}{l}\text { Sanches PG } \\
\text { Carvalho MD } \\
\text { de B }\end{array}$ & $\begin{array}{l}\text { Compreender como os enfermeiros } \\
\text { vivenciam o processo de morte e o } \\
\text { morrer dos pacientes }\end{array}$ \\
\hline XIV & $\begin{array}{l}\text { Cuidando do paciente no } \\
\text { processo de morte na } \\
\text { Unidade } \\
\text { de Terapia Intensiva }\end{array}$ & $\begin{array}{l}\text { Revista da } \\
\text { Escola } \\
\text { de } \\
\text { Enfermagem } \\
\text { da USP }\end{array}$ & 2011 & Pesquisa & $\begin{array}{l}\text { Silva RS da } \\
\text { Campos ERA } \\
\text { Pereira A }\end{array}$ & $\begin{array}{l}\text { Caracterizar o desenvolvimento do } \\
\text { cuidar/cuidado de enfermagem } \\
\text { numa } \\
\text { UTI ao paciente fora de possibilidade } \\
\text { de cura por enfermeiros. }\end{array}$ \\
\hline
\end{tabular}

Quadro 1 - Variáveis utilizadas para análise das publicações - Rio Grande, RS - 2012 conhecer a percepção de profissionais enfermeiros sobre a prática da ortotanásia no contexto hospitalar.

Conhecer os sentimentos vivenciados por esses trabalhadores diante do processo de morrer e morte de pacientes internados na unidade de terapia intensiva, bem como os mecânismos utilizados para o enfrentamento dessa questão. compreender como enfermeiras de UTIs vem enfrentando a aplicação de medidas terapêuticas que reconhecem como fúteis. 


\begin{tabular}{ll}
\hline $\begin{array}{l}\text { Necessidade de ações educativas } \\
\text { para a equipe de enfermagem }\end{array}$ & I, II, III, IV, V, VI, VII, VIII, \\
$\begin{array}{l}\text { Cuidados paliativos no processo } \\
\text { de morte e morrer }\end{array}$ & I, III, IX, X, XII, XIV \\
$\begin{array}{l}\text { Mecanismos de defesa no } \\
\text { processo de morte e morrer }\end{array}$ & I, IV, V, VII, XI, XIII \\
$\begin{array}{l}\text { Comunicação com o paciente e } \\
\text { a família no processo de morte e } \\
\text { morrer }\end{array}$ & I, II, III, VI, X, XIV \\
$\begin{array}{l}\text { Humanização no processo de } \\
\text { morte e morrer }\end{array}$ & II, III, V, VII, X, XII \\
$\begin{array}{l}\text { Uso da tecnologia no processo de } \\
\text { morte e morrer }\end{array}$ & II, IV, V, IX, XII, XIV \\
$\begin{array}{l}\text { Questões éticas e bioéticas } \\
\begin{array}{l}\text { Vinculação entre conhecimento } \\
\text { cientifico e a prática assistencial }\end{array}\end{array}$ & I, IX \\
\hline
\end{tabular}

Quadro 2- Enfoques apresentados pelos artigos - Rio Grande, RS - 2012

A partir dos enfoques identificados, foram elaboradas três categorias, apresentadas na discussão dos resultados: prática como objeto de reflexão e mudança; preparo profissional e instrumentalização;desafios éticos frente o processo de morte/morrer.

\section{DISCUSSÃO DOS RESULTADOS PRÁTICA COMO OBJETO DE REFLEXÃO E MUDANÇA}

Os cuidados paliativos são cada vez mais abordados nas questões relacionadas aos pacientes em processo de morte e morrer, preconizando-se o cuidado humanizado e individualizado. Evidencia-se a necessidade de profissionais que saibam incorporar em sua prática a filosofia dos cuidados paliativos, bem como com a morte e seus significados, como referido pelo artigo IX. Porém é salientado pelos profissionais de enfermagem a necessidade de aperfeiçoamentos e capacitações, sob a visão do tratamento paliativo, como é exposto nos artigos I, III, XII E XIV.

Ainda no que diz respeito a prática cotidiana, a comunicação com o paciente e a família no processo de morte e morrer é destacada como uma ferramenta extremamente importante em várias dimensões do cuidado de enfermagem. O apoio aos familiares desses pacientes é um ponto muito importante na assistência, dessa forma a enfermagem necessita estar pronta para lidar com sentimentos da família direcionados a equipe, como raiva e rancor, referenciado pelos artigos $\mathrm{VI}$ e X.

Em relação emprego da tecnologia durante o processo de morte morrer, destaca-se que esta representa progresso, resultando em importantes recursos para a manutenção da vida, por meio de diagnósticos e tratamentos mais precisos, como especificado pelo artigo IX. Porém, a tecnologia pode ser cotidianamente vista como aliada ou vilã, sendo assim, os artigos IV e XIV destacam que o emprego de medidas tecnológicas devem ser utilizados para auxilio no tratamento e não, para o reforço impensado do paradigma da cura.

\section{PREPARO PROFISSIONAL E INSTRUMENTALIZAÇÃO}

O cuidado está relacionado ao preenchimento das necessidades humanas básicas, proporcionando uma morte digna e tranqüila, quando a recuperação não for mais possível. Entretanto, o foco da humanização é a resolutividade e o direcionamento das ações para promoção do bem-estar do paciente terminal, como citado no artigo III. Embora os estudos II, III, V, VII, X e XII tenham abordado o tema, os mesmos restringiram-se a citações e caracterizações, avançando pouco no que diz respeito a estratégias mais concretas.

A busca do conhecimento alavancou o número de produções cientificas direcionadas as de situações geradoras de estresse frente o processo de morte e morrer, com isso a vinculação entre conhecimento cientifico e a prática assistencial, citada nos artigos I e IX, tornou-se fundamental na prestação do cuidado. Os artigos I, IV, V e VIII, destacam a importância de um aprimoramento continuado e uma formação especializada, com o objetivo de melhor preparar os profissionais para seus cotidianos de trabalho.

\section{DESAFIOS ÉTICOS FRENTE O PROCESSO DE MORTE/MORRER}

Devido ao contexto que envolve o processo de morte/ morrer e toda sua complexidade, as UTI's tornaram-se alvo de constantes conflitos éticos. Os estudos indicam a limitação das intervenções, como destaca o artigo X, e o emprego de medidas tecnológicas, como destaca o artigo IX. Além disso, outros fatores são geradores de conflitos, como as questões culturais, religiosas e sociais das pessoas envolvidas na equipe de saúde, destacados pelos artigos VIII e IX.

$\mathrm{O}$ artigo XII retrata o desafio ético de avaliar as medidas terapêuticas que devem ser utilizadas frente o processo de morte/morrer, preocupando-se com o indivíduo doente, respeitando sua integralidade sem esquecer que o cuidado é a base da enfermagem. $O$ artigo IX salienta que estes conflitos continuarão existindo, pois a cada dia os profissionais deparam-se com novas situações e tecnologias, reforçando a presença e atitude crítica do enfermeiro como vitais para uma assistência ética.

\section{CONSIDERAÇÕES FINAIS}

A prática profissional, o preparo para atuar frente o processo de morte/morrer e os desafios éticos são os grandes temas que convergem a produção científica de enfermagem sobre morte/morrer nos ambientes de UTI. Percebe-se a necessidade de investir na formação e no preparo de profissionais para atuarem no processo de morte/morrer, refletindo sobre suas práticas cotidianas, tornando-se mais conscientes da filosofia dos cuidados paliativos, assim como dos desafios éticos a serem enfrentados.

Destaca-se que a produção científica sobre a temática morte/morrer deve ser redirecionada na enfermagem 
brasileira, uma vez que a frequência de estudos descritivos e exploratórios, buscando o universo dos significados e sentimentos já se mostra bastante consistente e até mesmo desgastada. A construção de estratégias de enfrentamento, de tecnologias de cuidado e de formas de intervenção devem ser objetivadas em novas pesquisas mais conscientes com a realidade e necessidades, favorecendo assim pacientes, familiares e a própria equipe de enfermagem.

\section{Referências}

1. Menezes RA. Em busca da boa morte: antropologia dos cuidados paliativos. Rio de Janeiro: FIOCRUZ; 2004.

2. Pessini L, Barchifontaine CP. Problemas atuais de bioética. $7^{\text {a }}$ ed. São Paulo: Centro Universitário São Camilo; 2005.

3. Fugulin FMT, Rossetti AC, Ricardo CM, Possari JF, Mello MC, Gaidzinski RR. Tempo de assistência de enfermagem em unidade de terapia intensiva: avaliação dos parâmetros propostos pela resolução COFEN no 293/04. Rev. LatinoAm. Enfermagem. 2012;20(2):[09 telas].

4. Ribeiro KV, Soares MCS, Gonçalves CC, Medeiros IRN, Silva G. Eutanásia em paciente terminal: concepções de médicos e enfermeiros intensivistas. Enferm Foco. 2011;2(1):28-32.
5. Lima MGR, Nietsche EA, Teixeira JA. Reflexos da formação acadêmica na percepção do morrer e da morte por enfermeiros. Rev. Eletr. Enf. 2012;14(1):181-8.

6. Penha RM, Silva MJP. Significado de espiritualidade para enfermagem em cuidados intensivos. Texto Contexto Enferm. 2012;21(2):260-8.

7. Mendes KDS, Silveira RCCP, Galvão CM. Revisão Integrativa: Método de pesquisa para a incorporação de evidências na saúde e na enfermagem. Texto Contexto Enferm. 2008;17(4):758-64.

8. Cooper HM. Scientific guidelines for conducting integrative research reviews. Rev educ res. 1982; 52(2): 291-302.

9. Galiazzi MC, Moraes R. análise textual discursiva. Ijuí: Unijuí; 2011. 